\title{
Intravascular ultrasound chirp imaging
}

\author{
D. Maresca, ${ }^{1, a)}$ K. Jansen, ${ }^{1,2}$ G. Renaud, ${ }^{1}$ G. van Soest, ${ }^{1}$ X. Li, ${ }^{3}$ Q. Zhou, ${ }^{3}$ N. de Jong,,${ }^{1,2,4}$ \\ K. K. Shung, ${ }^{3}$ and A. F. W. van der Steen ${ }^{1,2}$ \\ ${ }^{1}$ Biomedical Engineering, Thorax Centre, Erasmus MC, 3000 CA Rotterdam, the Netherlands \\ ${ }^{2}$ Interuniversity Cardiology Institute of the Netherlands, 3501 DG Utrecht, the Netherlands \\ ${ }^{3}$ Department of Biomedical Engineering, University of Southern California, Los Angeles, California 90089, USA \\ ${ }^{4}$ Imaging Science and Technology, Delft University of Technology, 2628 CJ Delft, the Netherlands
}

(Received 1 November 2011; accepted 4 January 2012; published online 25 January 2012)

\begin{abstract}
We demonstrate the feasibility of intravascular ultrasound (IVUS) chirp imaging as well as chirp reversal ultrasound contrast imaging at intravascular ultrasound frequency. Chirp excitations were emitted with a $34 \mathrm{MHz}$ single crystal intravascular transducer and compared to conventional Gaussian-shaped pulses of equal acoustic pressure. The signal to noise ratio of the chirp images was increased by up to $9 \mathrm{~dB}$ relative to the conventional images. Imaging of contrast microbubbles was implemented by chirp reversal, achieving a contrast to tissue ratio of $12 \mathrm{~dB}$. The method shows potential for intravascular imaging of structures in and beyond coronary atherosclerotic plaques including vasa vasorum. (ㅇ 2012 American Institute of Physics. [doi:10.1063/1.3679375]
\end{abstract}

Atherosclerosis is a chronic systemic disease of the arterial wall and a leading cause of premature death worldwide. Most cardiac adverse events result from the rupture of a vulnerable atherosclerotic plaque and subsequent thrombosis in the coronary vascular tree. ${ }^{1}$ It is recognized that the formation of new microvessels in the arterial wall is critical to the progression of plaques due to red blood cell leakage and constitutes an important marker of plaque vulnerability. ${ }^{2}$ These networks of microvessels, ranging in diameter from 20 to $100 \mu \mathrm{m}$, are referred to as vasa vasorum (VV).

As of today, there are no clinically available tools for detecting VV in coronary artery plaques. Goertz et $a l^{3}$ reported the feasibility of VV imaging in animals by using an experimental intravascular ultrasound (IVUS) catheter and micron sized bubbles as ultrasound contrast agent (UCA) to reveal microvasculature which lies below the resolution limit. The method relied on the strong nonlinear response of a high concentration bolus of UCA compared to tissue.

In this letter, we develop chirp IVUS, with the dual objective of extending the viewing depth, and implementing chirp reversal contrast imaging in a manner that is applicable on a conventional IVUS catheter. At a given mechanical index (MI) and frequency bandwidth, chirp excitations carry more energy than conventional imaging pulses. As a result, chirp images display an extended tissue penetration depth. To date, ultrasound chirp imaging was implemented in noninvasive clinical imaging only. ${ }^{4}$ The transient resonant response of microbubbles close to resonance differs for insonification with up and down frequency sweeps. ${ }^{5,6}$ Capitalizing on this asymmetry, we present a contrast imaging mode that provides good contrast to tissue ratio (CTR) at clinically approved concentrations of UCA.

We investigated the performance of IVUS chirp imaging on different phantoms as well as an atherosclerotic human coronary artery ex vivo. Contrast chirp reversal imaging was evaluated using a channel phantom perfused with a commercial UCA.

\footnotetext{
${ }^{a)}$ Electronic mail: d.maresca@erasmusmc.nl.
}

The experimental intravascular imaging system consisted of a mechanically rotated single element IVUS transducer (single-crystal lead magnesium niobate-lead titanate; PMN$\mathrm{PT}^{7}$ active surface $0.5 \times 0.5 \mathrm{~mm}^{2}$, center frequency $34 \mathrm{MHz}$, $-6 \mathrm{~dB}$ bandwidth $60 \%$, and $2 \mathrm{~mm}$ natural focus) mounted in a catheter assembly with a $0.9 \mathrm{~mm}$ outer diameter. Interleaved Gaussian envelope sine wave pulses and chirp excitations of equal peak negative pressure were transmitted using an arbitrary waveform generator (Tabor Electronics WW2571A) at $1^{\circ}$ angular position steps using a motorized rotary stage (Steinmeyer $\mathrm{GmbH})$. The pulses were emitted at the transducer center frequency with a $60 \%-6 \mathrm{~dB}$ bandwidth. The chirp signal $s(t)$ can be formulated as $s(t)=a(t) \cos (2 \pi \varphi(t))$ where $a(t)$ is the envelope and the quasi-linear phase $\varphi(t)$, optimized to minimize the side lobe level, is defined in relation to the instantaneous frequency $f_{i}(t)$ as

$$
f_{i}(t)=\frac{1}{2 \pi} \frac{d \varphi(t)}{d t}=f_{1} \beta^{t} \quad \text { with } \quad \beta=\left(\frac{f_{2}}{f_{1}}\right)^{\frac{1}{T}}
$$

where $f_{1}$ is the initial chirp frequency, $f_{2}$ is the final chirp frequency, and $T$ is the signal duration. All chirp signals were apodized using an $11 \%$ tapered Tukey window. The ultrasound radio-frequency (RF) data were digitized at $350 \mathrm{MHz}$ (Acqiris DP-310) and band-pass filtered. The received chirp RF lines were compressed by cross-correlation with the emitted chirp signal.

The axial resolution and signal to noise ratio (SNR) were determined from echoes of $15 \mu \mathrm{m}$ thick platinum/iridium wires at distances of $1.5,3,5$, and $7 \mathrm{~mm}$ from the probe, mounted in a hollow cylinder. Additionally, a cylindrical agar-based phantom, ${ }^{8}$ with a lumen of $3 \mathrm{~mm}$ diameter and three parallel $2 \mathrm{~mm}$ channels at distances of 3,5 , and $7 \mathrm{~mm}$ off-axis, was designed in order to assess tissue penetration depth. Intravascular ultrasound chirp imaging of human coronary atherosclerosis was performed ex vivo. The artery was provided by the Pathology Department of the Erasmus Medical Center according to local Medical Ethics Committee regulations. The tissue was snap frozen at $-80{ }^{\circ} \mathrm{C}$ within an hour 


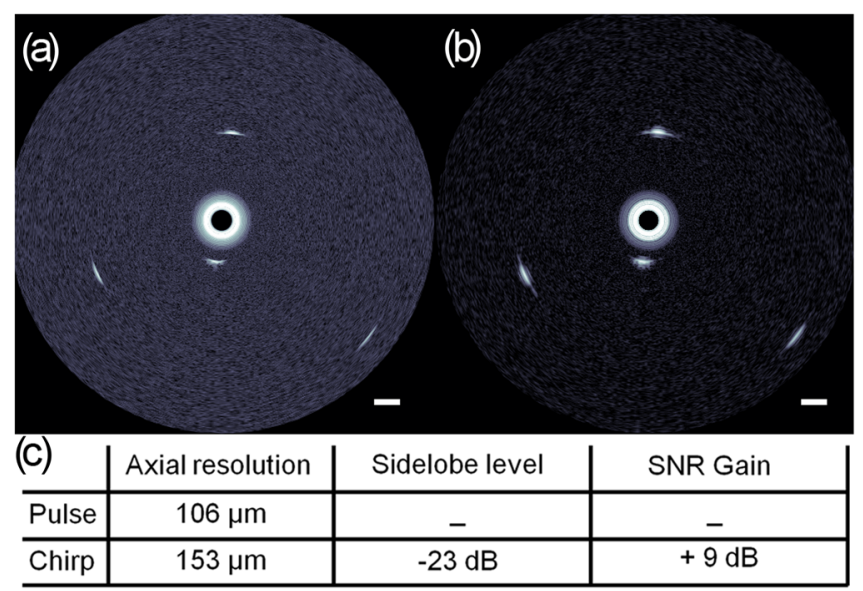

FIG. 1. (Color online) Subwavelength wire phantom imaged using pulses (a) and chirps (b). The dynamic range is $55 \mathrm{~dB}$. The scalebar represents $1 \mathrm{~mm}$. (c) Comparative performance of conventional IVUS versus chirp IVUS.

after autopsy. Before imaging, the specimen was thawed in phosphate buffered saline (PBS), mounted in a PBS filled tank, and pressurized to $100 \mathrm{mmHg}$ to keep the lumen open.

The chirp excitations used for the wire phantom measurement, shown in Fig. 1, had a temporal length of $0.3 \mu \mathrm{s}$, a frequency sweep ranging from 25 to $42.5 \mathrm{MHz}$ and an $\mathrm{MI}$ of 0.19. Using chirps, we measured a gain in SNR of $8 \mathrm{~dB}$ for the closest wire and $9 \mathrm{~dB}$ for the other three compared to the conventional imaging pulse. The axial resolution $(-6 \mathrm{~dB}$ point spread function) at the wire closest to the focus was $106 \mu \mathrm{m}$ for the pulse and $153 \mu \mathrm{m}$ for the chirp excitation after compression while the lateral resolution was $272 \mu \mathrm{m}$ for both. The sidelobe level in the compressed chirp image was at $-23 \mathrm{~dB}$ relative to the main lobe. Chirp imaging was applied to the tissue-mimicking phantom and compared to the pulse image, as displayed in Figs. 2(a) and 2(b). The third side channel appears at 8 o'clock in the chirp image only. Based on the SNR gain of $8 \mathrm{~dB}$, an imaging depth extension of $1.9 \mathrm{~mm}$, from $5.6 \mathrm{~mm}$ to $7.4 \mathrm{~mm}$ was achieved. With the ex vivo human coronary artery, a gain in SNR of $7 \mathrm{~dB}$ was measured in the chirp image compared to the pulse image (Figures 2(c) and 2(d)).

Chirp based contrast imaging was evaluated for its potential to detect VV. The UCA used was Definity ${ }^{\circledR}$ (Lantheus Medical Imaging, North Billerica, MA). We first simulated the pressure scattered by a $1 \mu \mathrm{m}$ diameter bubble in response to an up and down sweep chirp (duration $0.5 \mu \mathrm{s}$, frequency sweep 22 to $46 \mathrm{MHz}$, MI 0.19) with a visco-elastic shell model using the UCA high frequency shell parameters as described by de Jong. ${ }^{9}$ Next, for the experiment, the UCA was activated and decanted for 15 to $30 \mathrm{~min}$ to extract the smallest contrast bubbles which resonate at IVUS frequencies. With this method, the mean bubble diameter was less than $2 \mu \mathrm{m} .{ }^{10}$ Finally, a 1:1000 dilution was prepared. Chirp reversal contrast measurements were performed by filling the lumen and the first two channels of the tissue mimicking phantom with the UCA while the third one was filled with water. A two step pulsing scheme excited the UCA, first, with a down sweep chirp and then with an up sweep chirp transmitted at $10 \mu$ s intervals.

The simulation results clearly show the asymmetry between the bubble echoes of a down and up sweep chirp
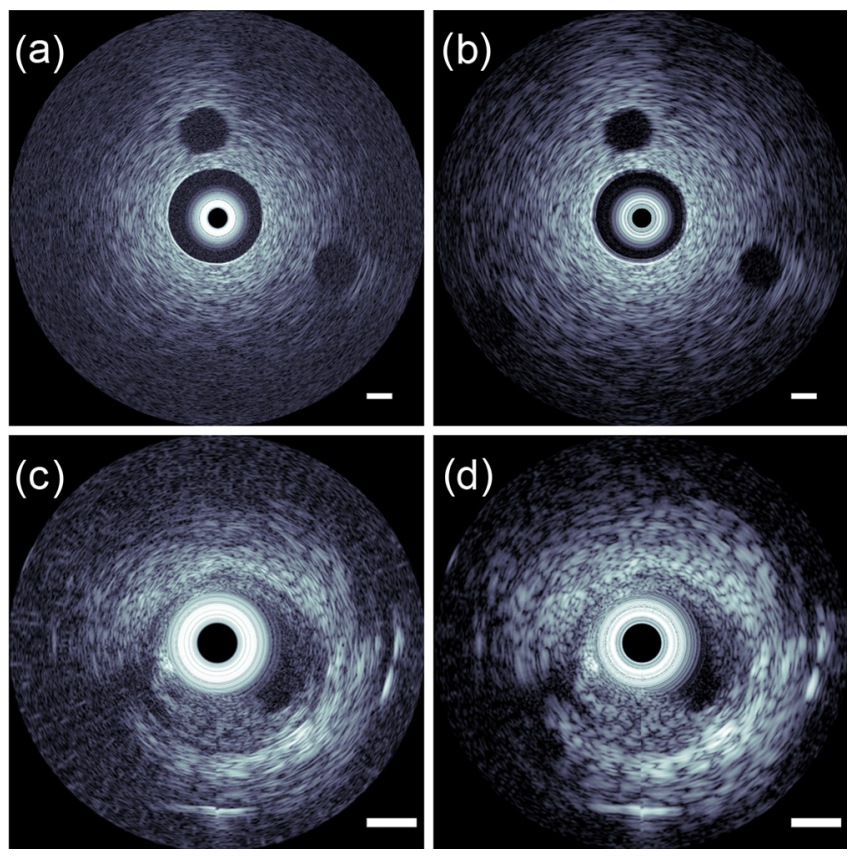

FIG. 2. (Color online) Channel phantom image using pulses (a) and chirps (b). The dynamic range is $55 \mathrm{~dB}$. Human coronary artery image using pulses (c) and chirps (d). The dynamic range is $48 \mathrm{~dB}$. The scalebar corresponds to $1 \mathrm{~mm}$.

(Figure 3(c) and 3(d)). A one micron diameter UCA bubble resonates at $25 \mathrm{MHz} .{ }^{10}$ The bubble response is enhanced when the driving frequency is close to its resonance frequency, which happens at the beginning for the up sweep chirp and at the end for the down sweep chirp. In addition, a ringdown effect elongates the bubble response to the down sweep chirp compared to the driving excitation. Therefore, the subtraction of the compressed bubble echoes gives a residual contrast signal. Experimentally, the chirp reversal contrast image had a CTR of $12 \mathrm{~dB}$ (ratio of mean bubble
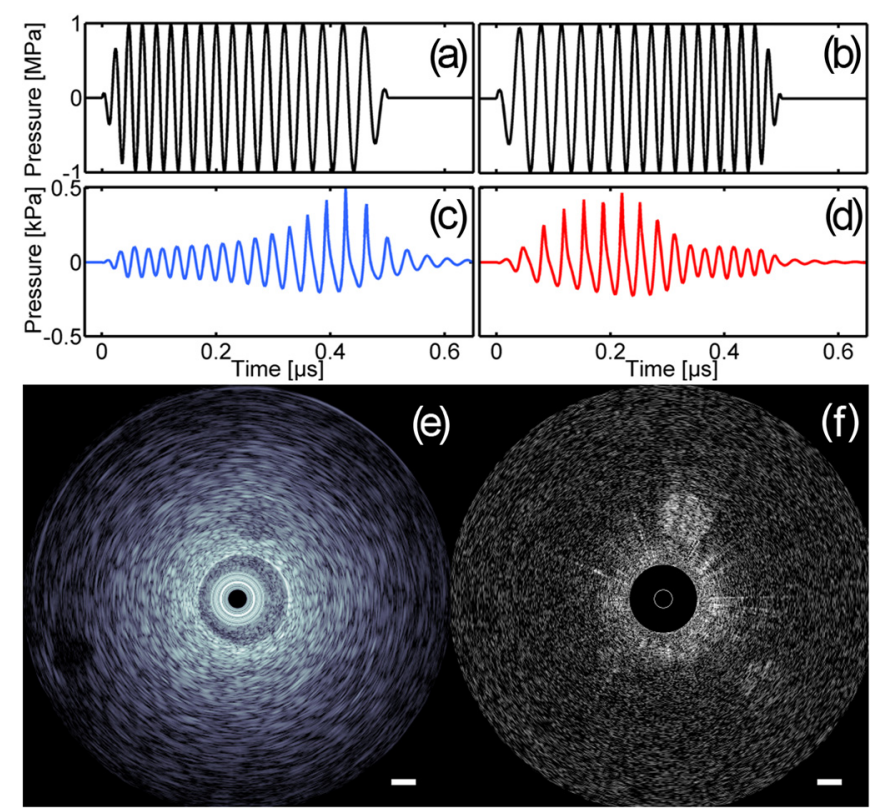

FIG. 3. (Color online) Simulated incident down (a) and up sweep (b) chirps sent a micron size UCA bubble and corresponding scattered acoustic pressures (c) and (d). (e) Down sweep chirp image and (f) contrast image of the channel phantom perfused with the UCA. The dynamic range is $54 \mathrm{~dB}$ for the left image and $21 \mathrm{~dB}$ for the contrast image. 
and tissue signal amplitudes) achieved at clinically approved concentration of this UCA (Figures 3(f)). Bubbles could be detected up to a depth of $5 \mathrm{~mm}$.

These results demonstrate the feasibility of intravascular ultrasound chirp imaging of human coronary atherosclerosis at intravascular ultrasound frequencies. A significant SNR gain was achieved, leading to an extension of signal penetration of $1.9 \mathrm{~mm}$, at some cost in axial resolution and limited appearance of side lobes. High-frequency chirp reversal contrast imaging was achieved. This IVUS contrast mode has the potential to image VV in a clinical setting, using commercially available UCAs in a low dose. Reliable in vivo quantification of VV holds promise for plaque vulnerability imaging.

The gain in SNR for coded excitations is equal to the time-bandwidth product, and as a result, chirp imaging benefits from long pulses. The duration of chirp excitations in IVUS is limited, however, because image targets are immediately adjacent to the transducer (Figures 2(c) and 2(d)). Theoretically, the SNR can be further enhanced, and the sidelobe level can be reduced, using mismatched filtering. ${ }^{11}$ IVUS is the clinical standard to assess the geometry and size of coronary plaques in vivo. Image interpretation and segmentation, often ambiguous tasks for trained evaluators at present, may benefit from the SNR gain, allowing for sharper contour detection.

Chirp IVUS will complement conventional IVUS methods by providing extended imaging depth, allowing for imaging deep into and beyond the plaque. Using chirp reversal, it adds a promising method for contrast-enhanced IVUS, imaging plaque vulnerability by quantifying plaque microvessels with low dose clinically approved UCAs, and with conventional frequency-band limited IVUS catheters.

${ }^{1}$ J. A. Schaar, J. E. Muller, E. Falk, R. Virmani, V. Fuster, P. W. Serruys, A. Colombo, C. Stefanadis, S. W. Casscells, P. R. Moreno, A. Maseri, and A. F. W. van der Steen, Eur. Heart J. 25(12), 1077 (2004).

${ }^{2}$ J. B. Michel, R. Virmani, E. Arbustini, and G. Pasterkamp, Eur. Heart J. 32(16), 1977 (2011).

${ }^{3}$ D. E. Goertz, M. E. Frijlink, D. Tempel, L. C. van Damme, R. Krams, J. A. Schaar, F. J. Ten Cate, P. W. Serruys, N. de Jong, and A. F. van der Steen, Invest. Radiol. 41(8), 631 (2006).

${ }^{4}$ J. Mamou, O. Aristizábal, R. H. Silverman, J. A. Ketterling, and D. H. Turnbull, Ultrasound Med. Biol. 35(7), 1198 (2009).

${ }^{5}$ A. Novell, S. van der Meer, M. Versluis, N. de Jong, and A. Bouakaz, IEEE Trans. Ultrason. Ferroelectr. Freq. Control 56(6), 1199 (2009).

${ }^{6}$ D. H. Thomas, P. Looney, R. Steel, N. Pelekasis, W. N. McDicken, T. Anderson, and V. Sboros, Appl. Phys. Lett. 94(24), 243902 (2009).

${ }^{7}$ Q. F. Zhou, X. C. Xu, E. J. Gottlieb, L. Sun, J. M. Cannata, H. Ameri, M. S. Humayun, P. D. Han, and K. K. Shung, IEEE Trans. Ultrason. Ferroelectr. Freq. Control 54(3), 668 (2007).

${ }^{8}$ C. J. P. M. Teirlinck, R. A. Bezemer, C. Kollmann, J. Lubbers, P. R. Hoskins, P. Fish, K. E. Fredfeldt, and U. G. Schaarschmidt, Ultrasonics 36(1-5), 653 (1998).

${ }^{9}$ N. de Jong, L. Hoff, T. Skotland, and N. Bom, Ultrasonics 30(2), 95 (1992).

${ }^{10}$ D. E. Goertz, N. de Jong, and A. F. van der Steen, Ultrasound Med. Biol. 33(9), 1376 (2007).

${ }^{11}$ T. Misaridis and J. A. Jensen, IEEE Trans. Ultrason. Ferroelectr. Freq. Control 52(2), 177 (2005). 